\title{
CONTINUIDADES Y CONTRAPUNTOS SOCIOPOLÍTICOS ENTRE DOS GENERACIONES DE DIRIGENTES ESTUDIANTILES DE LA UNIVERSIDAD DE LOS LAGOS
}

\author{
MARTÍN QUINTANA ${ }^{1}$ \\ FELIPE SARAVIA ${ }^{2}$ \\ Claudio VARGas ${ }^{3}$ \\ CÉSAR PÉREZ ${ }^{4}$
}

\begin{abstract}
RESUMEN
A partir de 15 entrevistas semiestructuradas, se contrastan los discursos de dos generaciones de dirigentes estudiantiles de la Universidad de Los Lagos (la de finales de los '80 y la actual), analizando cambios y continuidades en cuanto a épica, modos de organización y vínculos internos y externos. Los resultados muestran el tránsito desde una épica basada en proyectos políticos nacionales a otra reñida entre el pragmatismo y la subjetivación como colectivización de lo individual. Además, considerando su emplazamiento regional, ambas generaciones plantean contrapuntos respecto de la relación con el gobierno y las dirigencias metropolitanas, esbozando una mirada desde la descentralización.
\end{abstract}

PAlabRas ClaVe: DIRIGENTES ESTUDIANTILES, JÓVENES UNIVERSITARIOS, REgIÓN DE LOS LAGOS.

${ }^{1}$ Profesor de Estado en Castellano (Universidad de Los Lagos); Master en Cs Humaines et sociales (Université de Poitiers, France). Académico del Dpto. de Educación, Universidad de Los Lagos. Correo electrónico: m.quintana@ulagos.cl.

2 Trabajador social (Universidad Santo Tomás). Magíster en desarrollo humano a escala local y regional (Universidad de la Frontera). Candidato a doctor en ciencias sociales en estudios territoriales (Universidad de Los Lagos). Académico del Departamento de Ciencias Sociales de la Universidad del Bío-Bío. Correo electrónico: fsaravia@ubiobio.cl.

${ }^{3}$ Trabajador social (Universidad de Los Lagos). Estudiante del Magíster en Desarrollo Humano a Escala Local y Regional (Universidad de la Frontera). Becario CONICYT. Correo electrónico: clas.vargas@gmail.com.

${ }^{4}$ Egresado de antropología (Universidad Austral de Chile). Correo electrónico: kurvduam@gmail.com. 


\title{
CONTINUIDADES E CONTRAPONTOS SOCIOPOLÍTICOS ENTRE DUAS GERAÇÕES DE LÍDERES ESTUDANTIS DA UNIVERSIDADE DE LOS LAGOS
}

\begin{abstract}
RESUMO
A partir de 15 entrevistas semiestruturadas, são contrastados os discursos de duas gerações de líderes estudantis da Universidade de Los Lagos (a geração do final dos anos 80 e a atual), analisando mudanças e continuidades em relação à épica, modos de organização e vínculos internos e externos. Os resultados mostram a transição de uma épica baseada em projetos políticos nacionais para outra renhida entre o pragmatismo e a subjetivação como coletivização do individual. Além disso, considerando sua localização regional ambas as duas gerações propõem contrapontos a respeito da relação com o governo e as lideranças metropolitanas, esboçando uma olhada desde a descentralização.
\end{abstract}

PAlaVRas-ChaVe: LÍDERES ESTUDANTIS, JOVENS UNIVERSITÁRIOS, REGIÃO DE LOS LAGOS.

\section{SOCIOPOLITICAL CONTINUITIES AND COUNTERPOINTS BETWEEN TWO GENERATIONS OF STUDENT LEADERS FROM UNIVERSIDAD DE LOS LAGOS}

\begin{abstract}
Based on 15 semi-structured interviews, the discourses of two generations of student leaders from Universidad de Los Lagos (the generation of the late 1980 s and the current one) are contrasted in order to analyze changes and continuities in terms of their rhetoric, organization modes and internal and external links. The results show the transition from a discourse based on national political projects to another discourse, which is at odds between pragmatism and subjectivation as a collectivization of the individual. In addition, concerning their regional location, both generations contrast their particular relationship with the government and metropolitan leaderships, outlining their views from decentralization.
\end{abstract}

KEYWORDS: STUDENT LEADERS, YOUNG UNIVERSITY STUDENTS, LOS LAGOS REGION. 
74 Continuidades y contrapuntos sociopolíticos entre dos generaciones de dirigentes estudiantiles de la Universidad de Los Lagos

\section{INTRODUCCIÓN}

EN ESTE ESTUDIO se contrastan dos periodos con características sociohistóricas distintas: la dictadura militar de fines de los 80 y la democracia actual, no exenta de sostenidos cuestionamientos públicos y privados. Otra distinción a relevar es la arquitectura del sistema de educación superior y la característica del estudiantado. En efecto, hoy a pesar de los inminentes y aún cuestionados cambios del sistema de educación superior, la presencia de universidades regionales, públicas y privadas, es más habitual que la que había en los años $80, \mathrm{y}$ de manera persistente el acceso a la educación superior se ha ido masificando, aumentando el número de estudiantes que constituyen primera generación familiar en acceder a formación terciaria. En este contexto, el presente artículo busca analizar comparativamente la experiencia de dos generaciones de dirigencia estudiantil en una universidad estatal del sur de Chile, teniendo a la vista las distancias históricas que las separan. A partir de sus discursos, se compara a la generación actual con aquella que vivenció en carne propia el fin de la dictadura y la conquista de la democracia, dando cuenta de las continuidades y cambios en cuanto a forma de organización y actuación en relación con el sistema universitario y las redes circundantes de sus organizaciones.

Considerando los cambios sociopolíticos que ha habido desde la década de los 80 y 90 hasta la actualidad, aquí nos proponemos indagar en los cambios y continuidades en las formas y actuaciones de las organizaciones de estudiantes universitarios, tanto en el eje histórico como en el territorial. Actualmente existiría una actitud menos temerosa de la protesta pública, ha habido un cambio en la épica que sustenta la acción política, así como cambios en las motivaciones y tácticas. Las razones detrás de ello, estarían vinculadas a la diferencia de contextos históricos, tanto en la política nacional (dictadura y su postrimería versus democracia actual) como en cambios organizacionales 
(conversión de instituto profesional a universidad) y tecnológicos. Se propone también que las dirigencias de las dos generaciones estudiadas presentan contrastes relevantes respecto de las dirigencias de la capital nacional, que les ubican en una posición de menor relevancia percibida en las dinámicas políticas nacionales, aunque dada su lejanía física respecto de la región metropolitana, que dificultaría el control de procesos políticos locales, éstas han contado con mayor libertad para desarrollar experiencias políticas innovadoras.

La universidad en análisis es la Universidad de Los Lagos (en adelante ULA), que tiene su campus central en la región homónima, específicamente en la ciudad de Osorno, ubicada a más de 900 kilómetros de la capital nacional. Fue creada por ley el año 1993 bajo el gobierno del Presidente de la República Patricio Aylwin, aunque existía ya desde el año 1965 como sede local de la Universidad de Chile y, tras la intervención de la dictadura militar al sistema de educación superior chileno, con la consecuente fragmentación de las universidades públicas, se encontraba operando desde 1981, como Instituto Profesional de Osorno (en adelante IPO).

En la actualidad, la ULA, heredera del IPO, es la única universidad estatal de la región de Los Lagos, y constituye una de las instituciones de mayor peso en la oferta universitaria regional y de mayor aporte a las capacidades endógenas de la región (Delamaza, 2013). En términos generales, esto responde no sólo a su labor formadora de capital humano calificado, sino también a su misión de contribuir a la construcción de una sociedad regional más equitativa y la formación de ciudadanos que aporten en dichos procesos (Universidad de Los Lagos, 2013).

En este contexto, resulta relevante indagar cómo han evolucionado las formas de organización estudiantil en una universidad como la descrita. La Universidad de Los Lagos está emplazada en una región de la zona sur-austral de Chile, alejada territorialmente del centro político-administrativo del país. Como lo menciona Maturana (2015), estas regiones presentan características 
76 Continuidades y contrapuntos sociopoliticos entre dos generaciones de dirigentes estudiantiles de la Universidad de Los Lagos

particulares, tales como la ausencia de conurbaciones de gran tamaño o metrópolis donde tradicionalmente se concentran las grandes universidades en Chile. Esto ha redundado en desigualdades territoriales entre estas instituciones asociadas a su origen y las condiciones para su funcionamiento, determinadas en gran parte por la dictadura (Améstica, Gaete y Llinas-Audet, 2014) y refrendada, en el caso de la Universidad de Los Lagos, por la ley de creación del año 1993. En dicha ley se expresa un principio de estricta autofinanciación, declarándose que el desarrollo institucional no podrá, bajo circunstancia alguna, constituir una carga al erario público (Universidad de Los Lagos, 2016).

A pesar de que la institución universitaria en Chile ha sido un espacio de incidencia en la política nacional, conformación de nuevos partidos políticos (Huneeus, 1973), e incluso generación de elites políticas de recambio (Lobos, 2014), es esperable que, dada la lejanía con el centro político-administrativo del país, la realidad de la ULA difiera a la de universidades como la Universidad de Chile y la Pontificia Universidad Católica de Chile, emplazadas en la capital nacional, o la Universidad de Concepción, ubicada en la segunda ciudad más grande del país.

Dichas universidades son hoy reconocidas como universidades de investigación y doctorados (Universitas: El Mercurio, 2015), y se concentran principalmente en las tres regiones en las que existen metrópolis en Chile: Metropolitana, Valparaíso y Bío Bío (Mac-Clure y Calvo, 2013). La excepción es la Universidad Austral de Chile, ubicada en Los Ríos, región que, escindiéndose de la otrora mayor región de Los Lagos, fue creada el año 2007 a raíz de fuertes demandas territoriales impulsadas predominantemente por la elite local (Delamaza, 2010).

Las diferencias mencionadas, creemos, dan lugar a formas distintas de organización en la realidad del sur-austral de Chile, así como distintos perfiles de los dirigentes estudiantiles. Describir dichas diferencias facilitará una mejor comprensión del fenómeno de la participación estudiantil universitaria nacional, 
que creemos tiene matices territoriales que no deben ser obviados. Ello resulta relevante en un contexto en el que, salvo algunas pocas excepciones (Carrasco, Carrasco y Guerra, 2014; Orellana, Bivort, Farías y Martínez, 2014), las investigaciones sobre la relación entre juventud y política en Chile, han abordado el asunto principalmente desde una perspectiva nacional sin consideración de especificidades regionales.

En primer lugar, el artículo hace una contextualización bibliográfica en torno a la relación entre juventud, participación política y la universidad como espacio de participación juvenil. Posteriormente, se describe el método utilizado, y se detallan y debaten los resultados, para finalmente plantear alguna síntesis del estudio e interrogantes que surgen del análisis y que pueden constituir una futura agenda investigativa.

\section{CAMbios generaCiONALES de la PARTICIPACión POLítiCA DE LOS JÓVENES EN CHILE Y LA UNIVERSIDAD COMO UN ESPACIO PARADIGMÁTICO}

La relación entre la juventud y la participación política en Chile ha sufrido cambios profundos a lo largo del tiempo (Fernández, 2000). Durante la década del 60 y parte del 70, el interés por la política era una característica común a la mayor parte de la sociedad chilena, en especial de los jóvenes (Huneeus, 1973). Sin embargo, luego del golpe militar se reprimió casi toda forma de expresión democrática y de participación social, aun cuando éstas emergieron como mecanismos de resistencia a pesar de los costos personales y colectivos que ello implicaba. Con el retorno de la democracia en la década del 90 se observa una merma en la participación en la política institucional de la población general y de los jóvenes en particular (Carrasco, 2010; INJUV, 2004; Garretón, 1991). Sin embargo, esto último puede ser interpretado no como una renuncia a lo 
78 Continuidades y contrapuntos sociopolíticos entre dos generaciones de dirigentes estudiantiles de la Universidad de Los Lagos

político, sino como el plegamiento hacia una determinada forma de hacer política post-dictadura, a una democracia representativa excesivamente burocratizada y jerarquizada, frente a la cual los jóvenes tenderían a generar nuevas prácticas políticas definidas por la participación equitativa, por el asambleísmo, la autogestión, el pluralismo y la culturalización de la política (Valenzuela, 2007), expresiones que a pesar de concentrar una mayor proporción de participación juvenil que las formas de participación política tradicionales, solo logran movilizar a pequeños grupos (Orellana et al, 2014), al menos de manera tradicional.

En este contexto, se aborda el asunto desde un enfoque generacional (Muñoz, 2011), entendiendo que el concepto de juventud no es una simple categoría demográfica, sino que se trata de un producto socialmente construido, lo que se corrobora en el hecho de que los límites de lo que se entiende por tal, han demostrado ser históricamente variables (Ghiardo, 2004). Por lo tanto, la definición de juventud cambiará en tanto la sociedad en su conjunto cambie. De esta manera los cambios en la juventud pueden constituir un indicador de cambios societales mayores (Fernández, 2000) en la medida que, en la lógica de Ortega y Gasset, se conciba una generación como una cohorte demográfica que ha sido expuesta a más o menos a las mismas condiciones históricas de vida y que, por lo tanto, comparte la misma sensibilidad vital opuesta a la generación previa y a la posterior, lo que define su misión histórica (Leccardi y Feixa, 2011).

El enfoque de generación propuesto por Ortega y Gasset enfatiza el hecho de la superposición y convivencia de generaciones en un determinado momento histórico. Sin embargo, de acuerdo a Leccardi y Feixa (2011), no explica cómo es que una generación entrante llega a desarrollar una conciencia histórica común que le permita llegar a posicionarse de forma hegemónica. En cambio, la propuesta de Mannheim propone que las generaciones pueden ser entendidas como el resultado de discontinuidades históricas. Es decir, se constituyen 
debido a "por una parte, la presencia de acontecimientos que rompen la continuidad histórica y marcan un antes y un después en la vida colectiva; y por otra, el hecho de que estas discontinuidades sean experimentadas por miembros de un grupo de edad en un punto formativo en el que el proceso de socialización no ha concluido, por lo menos en sus fases más cruciales, y cuando los esquemas utilizados para interpretar la realidad todavía no son rígidos por completo" (Leccardi y Feixa, 2011:17).

Desde este prisma, es posible concebir ciertos hitos históricos como demarcadores generacionales en Chile, con sus consecuentes implicancias políticas. Toro (2008) observa que el plebiscito de 1988 constituye uno de dichos hitos, marcando un antes y un después en cuanto a participación electoral, confianza en las instituciones y legitimidad percibida de la democracia. Demuestra que un análisis basado en categorías etarias convencionales no logra explicar suficientemente las diferencias en estos aspectos, y propone tres momentos histórico-políticos que se corresponden con tres generaciones: la generación que participó del plebiscito, la generación adulta que no alcanzó a participar en el plebiscito (o adultos-jóvenes), y los jóvenes. Éstas no sólo se diferencian en los aspectos propiamente políticos, sino también en aspectos culturales más generales, siendo más liberales los jóvenes que los adultos-jóvenes, y, a su vez, éstos más liberales que los adultos que participaron del plebiscito. Toro (2008) da cuenta de la particularidad de Chile en este sentido, al compararlo con el escenario latinoamericano en general, relativizando así la idea de que la juventud sería una etapa caracterizada por la inmadurez y que en edades más avanzadas necesariamente tendería a adoptar las actitudes adultas esperadas. Quizá un punto de tensión con estos planteamientos, puedan ser revisados a posteriori, matizando por ejemplo condiciones sociales de construcción de juventud en diferentes clases, culturas o territorios.

Así como el plebiscito marcó un hito en tanto épica para el retorno de la 
80 Continuidades y contrapuntos sociopolíticos entre dos generaciones de dirigentes estudiantiles de la Universidad de Los Lagos

democracia, diversos estudios dan cuenta también de la relevancia histórica del movimiento estudiantil de los años 2006 y 2011, que podría constituirse en un nuevo demarcador generacional, abriendo paso a lo que algunos autores han llamado "la generación sin miedo" (Cummings, 2015).

En efecto, lo ocurrido desde 2006 se diferencia del escenario anterior, ya que, con excepción de las protestas contra la ley marco de universidades estatales suscitadas en 1997 (Cifuentes, Cuenca y Salinas, 1998), las décadas de 1990 y del 2000 tuvieron pocas protestas estudiantiles. En contraste, en abril de 2006 los estudiantes secundarios dieron inicio a la más larga ola de protestas desde el retorno de la democracia, llegando a movilizar a más de un millón de personas a lo largo de todo el país (Cummings, 2015).

Ante el surgimiento de las movilizaciones desde el año 2006 se han propuesto varios factores explicativos. En primer lugar, un descontento o malestar más o menos generalizado en las generaciones más jóvenes (Mayol y Azocar, 2011), producido por la creciente brecha entre expectativas (atizadas por el éxito macroeconómico y desarrollo del neoliberalismo en el país) y posibilidades reales de alcanzarlas (Cummings, 2015), cuya disparidad es evidente dadas las condiciones de desigualdad económica nacional (OCDE, 2011), segregación escolar (Bellei, 2013; Waissbluth, 2010), altos costos de la educación superior (Marcel y Tokman, 2005), y reproducción de las desigualdades a pesar de la ampliación de la cobertura en educación terciaria (Núñez y Gutiérrez, 2004).

Ahora bien, como plantea Cummings (2015), el malestar es un factor necesario para el surgimiento de la protesta, pero no es el único. Otro factor mencionado por el autor es el hecho de que los movilizados nacieran en democracia por lo que se encontrarían libres del temor a la represión sufrida por las generaciones precedentes -salvo en caso de descendientes directos de víctimas de la represión de Estado en que es posible pensar que la memoria familiar opere como un cierto sostenedor del miedo o la rabia- facilitándose así 
la traducción del malestar en protesta.

Adicionalmente, Salinas y Fraser (2012) consideran que el movimiento estudiantil, cuyo foco estaba puesto en temáticas educacionales, es producto también de un conjunto de nuevas condiciones creadas por el desarrollo económico y expansión educacional vivida por el país en las últimas décadas, que configuraron una generación joven con mayor capacidades y actitudes críticas traducibles en acción colectiva. No obstante, esto no puede leerse de manera lineal, en tanto supondría que las generaciones precedentes carecían de estas condiciones, lo que no fue necesariamente así. En todo caso, este factor podría verse amplificado además por la expansión del acceso a la educación, donde José Joaquín Brunner registra un aumento de la cobertura de la población entre 18 y 24 años de edad desde un $15,6 \%$ en 1990 a un 38,3\% en el 2006 (Salinas y Fraser, 2012).

Dichas capacidades se encuentran asociadas también en parte, a la disponibilidad de nuevas tecnologías de la información y comunicación (en adelante TIC). En la generación de las movilizaciones del 2006 y 2011 se observa una diferencia radical respecto de las generaciones precedentes, ya que, como plantea Valderrama, "gran parte de los sujetos escolares del 2006 habían crecido durante la post-dictadura frente a pantallas, inmersos en un periodo de amplia inserción digital (...). Se habían apropiado de las tecnologías digitales, haciéndolas indispensables e indisolubles de sus procesos comunicativos y ciudadanos, como la forma casi natural de desenvolverse en el mundo" (2013, p.133).

Esta generación hizo uso de un conjunto de medios no tradicionales para complementar la protesta, tales como correo electrónico, mensajería instantánea y redes virtuales como Fotolog, Twitter y Facebook, así también el servicio de difusión de videos YouTube. Ello, sumado a acciones masivas no necesariamente digitales (aunque digitalizables) como flashmobs, "besatones", cuerpos pintados, entre otras, contribuyó a la masificación del movimiento, 
82 Continuidades y contrapuntos sociopolíticos entre dos generaciones de dirigentes estudiantiles de la Universidad de Los Lagos

generándose por parte del gobierno respuestas cuasi-coercitivas, como el anuncio del gobierno de Sebastián Piñera de monitorear la actividad virtual de Twitter, lo que despertó un repudio generalizado en la opinión pública nacional (Rodríguez, Peña, y Sáez, 2014).

En este sentido, citando al ex dirigente estudiantil Giorgio Jackson, Cabalín (2014) plantea que internet puede ser vista como un contrapeso al monopolio de los medios de comunicación de masas convencionales. No obstante, como argumentan Rodríguez, Peña, y Sáez (2014), el uso masivo de TIC en las movilizaciones en Chile no necesariamente ha redundado en una crítica coherente y de fondo a la concentración de los medios de comunicación en unos pocos conglomerados económicos, y a su homogeneidad ideológica.

Otro factor distintivo de la generación del 2006-2011 es mencionado por Donoso (2013), y tiene relación con el entorno político en el que emergen las protestas del 2006. La Concertación de Partidos por la Democracia se había caracterizado en las décadas de 1990 y del 2000 por desarrollar una forma de hacer política elitista (Delamaza, 2013b), que mantuvo los espacios de participación ciudadana más bien acotados, buscando mantener una cierta estabilidad política en un contexto de temores por la fragilidad de la democracia recuperada en 1990. Sin embargo, a mediados del 2000, estudios comienzan a dar cuenta de una disconformidad creciente de la ciudadanía con los resultados del modelo chileno. En dicho contexto, la campaña de la entonces candidata presidencial Michelle Bachelet efectúa un giro importante al enfatizar el carácter "ciudadano" de su programa y el estilo "bottom-up" de su agenda. De acuerdo a Donoso (2013), ello es visto como una ventana, una oportunidad política, por el movimiento estudiantil, posibilitándose así el rápido crecimiento de la movilización.

Debe considerarse también un factor contextual de carácter macroestructural, cual es la ampliación y diferenciación según cohortes de las clases medias durante las últimas décadas (Mac-Clure, 2012), siendo el movimiento una 
expresión de unas incipientes generaciones de clases medias (Fleet, 2011) con marcados objetivos aspiracionales, tal como lo indica Bajoit: "Ayer, el hijo -y menos aún su hija- del campesino, del obrero, del almacenero, del taxista... no se sentían llamados a ir a la universidad, y se conformaban con su destino social, sin vivirlo con frustración. Esto ha cambiado radicalmente en el curso de los últimos treinta años" (2016, p. 14). Esto queda reflejado también en los relatos de los discursos de los dirigentes del movimiento de los "pingüinos" (estudiantes secundarios) en el estudio realizado por Donoso (2013).

Los factores mencionados constituyen el trasfondo de lo que, a nuestro parecer, puede ser visto como un hito que marca a una generación de estudiantes secundarios en el caso de la movilización del 2006, y a universitarios durante el 2011. Sin embargo, cabe cuestionarse si esta generación trae consigo la conformación de una nueva identidad política colectiva. A este respecto, Bro (2011) plantea que, a pesar de que evidentemente hay un conjunto de transformaciones político-culturales expresadas particularmente en el "movimiento pingüino", estas no pueden ser consideradas como la emergencia de una nueva identidad política colectiva propiamente tal, sino como la conjunción de fuerzas sociales que no respondieron a determinadas ideologías, que inclusive lograron generar sinergias inter-ideológicas en función de un conjunto de fines más bien pragmáticos. En una de las entrevistas realizadas por el autor a estudiantes que participaron de las movilizaciones, se resalta el valor del individuo: "las banderas políticas son como las religiones, como que abarcan a una cachada de gente, pero ¿dónde queda el individuo? ¿Dónde queda la política del individuo? ¿Dónde queda la fe del individuo? no toda la gente de una misma religión cree en lo mismo" (Bro, 2011).

Esta "política del individuo" puede ser considerada entonces, una de las marcas diferenciadoras sustantivas del movimiento pingüino respecto de los movimientos estudiantiles de décadas pasadas, en la medida que como plantea 
84 Continuidades y contrapuntos sociopolíticos entre dos generaciones de dirigentes estudiantiles de la Universidad de Los Lagos

Bro (2011) uno de sus factores explicativos fundamentales radica en la disolución de categorías de comprensión de la sociedad totalizantes, que hace tiempo han desaparecido, y que, sin embargo, son tradicionalmente requeridas para la acción colectiva.

\section{MÉTODo}

Se optó por un análisis comprensivo a entrevistas realizadas a dirigentes estudiantiles de la Universidad de Los Lagos que hayan participado como Consejeros Universitarios, en la Federación de Estudiantes, Centros de Alumnos o en el Tribunal Calificador de Elecciones (TRICEL) de alguna carrera. Además, se incluyeron dirigentes de organizaciones estudiantiles no directamente políticas, tales como religiosas o sociales.

Se entrevistó en total a quince sujetos, de los cuales doce son dirigentes actualmente (al momento de la entrevista), y tres son ex dirigentes de la generación que vivenció el retorno a la democracia durante su experiencia dirigencial, así como el proceso de conversión del IPO en ULA. Estos tres tienen en común que trabajan actualmente como académicos en la misma universidad en la que fueron dirigentes estudiantiles y dos de ellos ocupan cargos de dirección central institucional. Por ello, no se pretende que su discurso sea necesariamente representativo de toda su generación, sino que más bien se busca generar contrapuntos entre participantes de una particular generación dirigencial del pasado, con la actual generación de dirigentes.

Las entrevistas fueron semiestructuradas, y se enfocaron en dos grandes dimensiones: a) caracterización de la organización representada; y b) vinculación y comunicación entre la organización y con otros actores tanto externos como internos a la comunidad universitaria. 
Las entrevistas fueron codificadas según las dimensiones y tópicos de interés del estudio, sometiendo el corpus discursivo a un proceso de ordenamiento y triangulación recursiva por diferentes investigadores. Este sistema permite arribar a un análisis más válido por cuanto se evita depender de la interpretación idiosincrática de un investigador único e individual. A partir del análisis de las coincidencias más fuertes entre los diferentes discursos, se licitaron ciertas líneas argumentales que dan respuesta al propósito del estudio.

\section{RESUlTADOS Y DISCUSIÓN}

A continuación, se da cuenta de los resultados del análisis, ordenándolos en función de dos ejes articuladores: un eje temporal en el que se enfatizan los contrapuntos, en términos de continuidades y rupturas, generados entre ambas generaciones, y un eje territorial en el que ambas generaciones establecen contrapuntos acerca de su posición socio-territorial respecto de las dirigencias estudiantiles de la Región Metropolitana.

\subsection{CONTRAPUNTOS TEMPORALES}

El análisis realizado da cuenta de cambios y al mismo tiempo permanencias entre las dirigencias de ambas generaciones. Ello es así en cuanto a la épica o imaginario motivacional detrás de la dirigencia estudiantil, así como en los modos de organización, los vínculos sostenidos al interior de la universidad entre distintas organizaciones estudiantiles y con las autoridades académicas, y también con los vínculos sostenidos con el entorno social y político externo a la universidad. 
86 Continuidades y contrapuntos sociopolíticos entre dos generaciones de dirigentes estudiantiles de la Universidad de Los Lagos

\subsection{1 ÉPICAS}

La decisión de adoptar para el análisis el concepto de épica, antes que otros tales como narración o relato, obedece a una comprensión del discurso político en constante relación con la agencia social.

La épica, conocida originalmente como epos, es objeto predilecto de los estudios sobre literatura, folclor y cultura popular que consiste en todo género de relatos heroicos y hazañosos, inicialmente de trasmisión oral y más tarde escritos, progresión que tiene que ver con el proceso de fijación de lo nacional popular, propio del nacionalismo moderno. Lo relevante aquí es su sentido político, que se puede decir que ocurre en dos movimientos generativos que concurren de manera simultánea. El primero tiene que ver con una concepción estructural del tiempo, propia del relato épico, diferente del tiempo cronológico, y según la cual se va instanciando una oposición simbólica entre el pasado mitificado y el presente que es vivido e imaginado tomando a aquél como referencia. En el otro movimiento, el sujeto se va conformando en el discurso narrado y en el mismo acto narrativo, de manera que también genera una oposición simbólica entre el nosotros y los otros. Así, a partir de estas dos instancias se gesta una relación mutua entre ethos y epos: identidad y épica (Gravano, 2016). El sujeto así concienciado adquiere el potencial de constituirse organizacionalmente, de actualizar su subjetividad en prácticas colectivas.

En razón de ello es que aquí lo épico debe entenderse en un sentido más materializable o más encarnado que el concepto de narrativa, dado que reconoce la capacidad de los colectivos de construir a través del lenguaje un concepto del mundo, de su funcionamiento, una categorización de los actores que habitan el espacio social, y el lugar, función y misión del propio colectivo (lo que implica constituirse como sujetos) dentro del mundo. En efecto, la épica 
es justamente aquello que permite la emergencia de una dialéctica entre narrativa y acción, y que, en definitiva, convoca a individuos y a grupos a constituirse bajo un sujeto político común. Y junto con este proceso de subjetivación (entendido aquí de manera más autoconsciente que, por ejemplo, en el habitus propuesto por Bourdieu), se realiza la objetivación de la subjetividad a través de la acción social que se coloca como condición la posibilidad de correr riesgos en función de un objetivo político.

El riesgo es crucial, en este concepto de lo épico, por cuanto implica una concienciación previa acerca de los peligros que entraña la acción orientada al cambio social. Hay que entender que el cambio social equivale a la ruptura del statu quo que es sacralizado en las diversas instancias rituales de la cultura, tanto en lo cotidiano como en lo periódico. Por lo tanto, en la épica se da un acto de conversión simbólica del peligro al riesgo. El peligro es lo inefable, lo no conmensurado y que, por su amenaza al estado de cosas y a la cultura, se instala como lo sagrado (Bergua, 2016). En tanto que el riesgo es la ruptura del tabú, el acceso consciente a lo sagrado para su manipulación. De esta manera, las épicas constituyen un dispositivo discursivo práxico, productor de una cultura que busca subvertir la cultura actual, tanto en su sentido simbólico (mundos, sujetos) como material (estructura social).

Los dirigentes antiguos perciben a la nueva generación estableciendo una solución de continuidad sustancial que, en su progresión hacia el presente, va experimentando ciertos cambios. En efecto, la nueva generación es representada por la antigua como la continuación natural de la lucha llevada a cabo en décadas anteriores: "van tras un objetivo que es el mismo que nosotros teníamos antes, que tiene otro nombre no más, a nosotros nos cambiaron la figura de Pinochet, pero el modelo permanece" (Presidente Federación de Estudiantes, generación 80-90).

Los discursos inmediatos de los dirigentes actuales son menos idealistas respecto de sus fines. Éstos obedecen más a objetivos específicos y pragmáticos 
88 Continuidades y contrapuntos sociopolíticos entre dos generaciones de dirigentes estudiantiles de la Universidad de Los Lagos

que a un gran proyecto político nacional. En general, tanto los representantes de Federación como de Centros de Estudiantes plantean sus objetivos asociados a generar instancias de fortalecimiento de las relaciones al interior de sus carreras, aportar al mejoramiento del currículum de sus compañeros, y el bienestar general del estudiantado.

Este pragmatismo se expresa incluso en el hecho de que la labor dirigencial es supeditada al tiempo disponible después de las actividades académicas formales. La labor dirigencial es vista en este contexto, como accesoria en contraste con las actividades académicas propias del ser estudiante universitario: "no puedes empeñarle todo el tiempo a eso, puesto que también eres estudiante, entonces tienes muchas veces una gran cantidad de aspiraciones, pero no la vas a poder cumplir todas" (Vicepresidente, Centro de Estudiantes Pedagogía en Matemática, generación actual).

Contrastando lo anterior, en el caso de los movimientos políticos existentes al interior de la universidad, como el FUR (Fuerza Universitaria Rebelde), la UNE (Unión Nacional Estudiantil), y la Izquierda Autónoma (IA), los fines declarados se asocian a dinamizar la vida social, cultural y política de la universidad, que en la actualidad es percibida como débil, lo que se asocia a los efectos profundos y de largo plazo de la instauración del modelo neoliberal en Chile:

"los últimos 25 años, el sistema neoliberal ha carcomido no solamente la educación, si no que la salud, el trabajo y la vivienda; ha llevado a la deconstitución de estos sujetos populares que antes se encontraban, hablemos de los años 70”. (Miembro FUR, generación actual)

En nuestra interpretación, los movimientos propiamente políticos generan, en mayor medida que los Centros de Estudiantes y Federación de Estudiantes, un discurso que podría considerarse la continuación de la lucha de la década del 80 y 90 en su sentido de articulación nacional. Se aprecia, de todos modos, una 
transformación de las épicas que movilizan la acción dirigencial.

En ambos momentos surge una crítica del dirigente universitario hacia la sociedad mayor y sobre la cual asienta su praxis sociopolítica. De una parte, la generación antigua adhirió y contribuyó a la épica de la recuperación de la democracia que tuvo alcance nacional, logrando articularse en convergencia histórica con otros sujetos y con otras estructuras político-dirigenciales a través de los partidos políticos. Por su parte, la épica de las dirigencias actuales también responde a un análisis del momento sociohistórico actual, concatenado a los cambios sociales producidos en décadas anteriores. La diferencia radica en que está más asociada a la vitalización del sujeto político, a la promoción de una sensibilidad política como condición previa a la política tradicional. El siguiente fragmento de entrevista es elocuente en este sentido:

yo creo que la principal característica que nos da es cómo nosotros vamos construyendo un tejido social amplio y que sea este tejido social amplio el que vaya decidiendo para después qué modelo de vida y qué modelo de sociedad quiere, porque, por ejemplo, no puedes llegar y decir "sabes, que queremos derrumbar el sistema capitalista", pero si no tienes una sociedad que lo quiera hacer, no tienes un acople con la realidad. La realidad es otra, la gente hoy en día quiere otra cosa, la gente es feliz prácticamente con el sistema que existe hoy en día, independiente de su extracción de clase que tenga. Por lo tanto, más que mejorar el vínculo, creo yo, hacer también ver a todas las organizaciones que están dentro de esta izquierda que se está conformando hace muchos años en Chile, es de entender que hoy en día no hay que replicar las lógicas de construcción que, por ejemplo, tuvieron la izquierda durante los años 90. Este objetivo del colectivo, este tema como de la vanguardia que aún se ve reflejada dentro de los movimientos sociales. (Miembro FUR, generación actual)

Otro aspecto relevante es que estas narrativas se construyen preferentemente desde espacios que tienen una articulación nacional o, al menos, extra-regional. 
90 Continuidades y contrapuntos sociopolíticos entre dos generaciones de dirigentes estudiantiles de la Universidad de Los Lagos

En la generación de los 80 y 90, el espacio era el partido político, mientras que actualmente, estas épicas son sostenidas de manera más explícita por los dirigentes que pertenecen a movimientos políticos extra-locales. Se puede argüir que la conformación de orgánicas que exceden lo local, suscitan y/o son suscitadas por narrativas que también son extra-locales y que dan lugar a épicas en las que los sujetos políticos se constituyen a sí mismo bajo los términos de su accionar histórico.

En coexistencia con esta épica más globalizante, en la actualidad se dan luchas que podrían ser consideradas más bien como luchas focalizadas, en el sentido de que están abocadas predominantemente al mejoramiento de condiciones específicas del estudiantado local.

\subsubsection{Modos DE ORganización Y RELEVANCIA DE LOS PARTIDOS POLÍTICOS}

Los contrastes entre las dos generaciones analizadas confirman lo planteado en diversos estudios (Madrid, 2005; Cheresky, 2006; Zarzuri, 2010) en relación con la perdida de centralidad de los partidos políticos tradicionales, y la emergencia de nuevas formas de organización asociadas a nuevas tecnologías de la comunicación e información. En el caso de la primera tesis, nos permite añadir que el poder de los partidos políticos se ha visto disputados por nuevas organizaciones políticas protopartidarias, en el caso del uso de las tecnologías, ha resignificado el modo en que se genera la organización y la participación social y política (Castells, 2012).

Las dirigencias estudiantiles de la generación de finales de los ochenta se caracterizaban por una fuerte y transversal influencia de partidos políticos tradicionales en las organizaciones, lo que es representado como un facilitador de las relaciones entre las distintas organizaciones al interior y exterior de la 
universidad, así como un importante espacio de formación política. En términos concretos, para dicha generación casi no era distinguible la labor como dirigente estudiantil, de la labor política partidista, aunque el devenir de los días fuera, en muchos casos, desalentando la militancia:

yo provenía de mi barrio en Santiago, allí yo era militante pero en la universidad no, porque me desencantó digamos la militancia que existía en ese minuto; entonces yo fui más bien simpatizante de algunos movimientos no convencionales, no tradicionales, más que militante. (Vicepresidente de Federación de Estudiantes, generación 80-90)

Los partidos que otrora fueran mecanismos útiles para los fines de las dirigencias estudiantiles, hoy son considerados como obsoletos, elitistas y poco democráticos. Ello ha implicado que hoy los movimientos políticos de izquierda se organicen de formas radicalmente distintas a las del pasado, pero también que las organizaciones estudiantiles sean conformadas por estudiantes que no necesariamente son militantes: "La federación está compuesta por diferentes integrantes de organizaciones, no todas, en este caso yo no pertenezco a ninguna organización y así otros no pertenecen a ninguna" (Presidente Federación de Estudiantes, generación actual).

Un aspecto relevante del cambio en los modos de organización dice relación con las jerarquías en las relaciones al interior de ellas. Mientras en la actualidad todos los dirigentes declaran establecer relaciones mayormente horizontales mediadas a través de Facebook, Twitter, y WhatsApp, en la generación de los 80 y 90 se establecían relaciones verticales a través de precarios medios de comunicación, en un contexto en el que dichas comunicaciones implicaban un peligro concreto: la dictadura y su intervencionismo dentro de la Universidad:

Las cuentas políticas semanales que el encargado del núcleo o de la célula, de la base, entregaba al grupo de compañeros, esos ocho o diez compañeros, y eso era algo bastante ritual, (...) venía en un papelillo, en un papelillo en papel de mantequilla, doblado en cuadritos y entonces se desplegaba ese 
92 Continuidades y contrapuntos sociopolíticos entre dos generaciones de dirigentes estudiantiles de la Universidad de Los Lagos

papelillo en cuadritos y el encargado de la base exponía lo que allí estaba, ese papelito había sido entregado por la dirección superior de ese compañero y a esa dirección superior se la había entregado la comisión política de la juventud comunista, y entonces era la orientación política de la semana o del mes. (Presidente Federación de Estudiantes, generación 80-90).

\subsubsection{VÍNCULOS AL INTERIOR DE LA UNIVERSIDAD}

En cuanto a los vínculos establecidos al interior de la universidad, los entrevistados se refieren principalmente a las relaciones con autoridades académicas y relaciones entre distintas organizaciones estudiantiles. Ambas han experimentado cambios.

Los entrevistados de la década del 80 y 90 valoran como signo de madurez política el haber transitado desde estrategias de incidencia mediante acciones de fuerza y presión a estrategias políticas más dialogantes con las autoridades académicas. En este sentido, se destaca como una conquista de la dirigencia estudiantil de esa época, la instalación de espacios democráticos que permitieron que la voz de los estudiantes sea escuchada por parte de las instancias directivas formales de la universidad, pero al mismo tiempo consideran que la generación actual no valora suficientemente esas conquistas del pasado:

Los espacios democráticos que se fueron conquistando de participación estudiantil, ellos no los consideran como tal, yo creo que ellos no valoran esos espacios, y se empoderan cuando le doblan la mano completa al sistema, cuando se toman la universidad, (...) pero cuando hay espacios de discusión democrática que nos ganamos también con conquista y con lucha, ellos no los valoran (...). (Vicepresidente de Federación de Estudiantes, generación 80-90) 
En efecto, los dirigentes actuales reconocen la situación planteada por la generación anterior, no solo en cuanto a las instancias formales en relación con las autoridades académicas, sino incluso en las instancias de participación al interior de las organizaciones estudiantiles, en las que difícilmente se logra una convocatoria amplia. Sin embargo, una de las razones que explicarían la débil participación en las instancias formales se asocia al carácter consultivo y no vinculante de la función de los estudiantes en ellas:

que estos espacios tengan un carácter consultivo en sí es un tremendo problema porque no está generando la cultura y la necesidad en el estudiante de poder tomarse en serio estos roles, (...) porque no ven en realidad mayor incidencia en estos espacios. (Representante estudiantil en consejo superior, generación actual)

Es decir, no es que las conquistas de las generaciones anteriores no sean valoradas, sino que no logran satisfacer las demandas actuales, más radicales y exigentes que las del pasado, puesto que plantean el requerimiento de una democracia universitaria más completa, profunda y vinculante.

Este discurso no necesariamente representa a la mayoría de los dirigentes actuales. En general la relación con las autoridades académicas es vista nuevamente en términos pragmáticos, asociada a actividades puntuales, como la organización de congresos académicos, seminarios, postulación de proyectos y la búsqueda de apoyo y financiamiento para realizar acciones de vinculación con el entorno. En este sentido, los miembros de movimientos políticos constituyen una minoría con una propuesta política que apunta más allá de lo pragmático, pero que no precisamente moviliza al conjunto de organizaciones estudiantiles.

En cuanto a la relación entre las organizaciones estudiantiles también se observan cambios. La generación de las décadas del 80 y 90 describe una universidad pequeña en la que las diversas agrupaciones y partidos políticos se relacionaban de manera estrecha, incluso al punto de flexibilizar en lo local 
94 Continuidades y contrapuntos sociopolíticos entre dos generaciones de dirigentes estudiantiles de la Universidad de Los Lagos

posturas nacionales, con el fin de generar fuerzas comunes en función de ciertas causas específicas. En la generación actual, en cambio, las relaciones entre las organizaciones estudiantiles parecen no ser muy estrechas, con excepción de los movimientos propiamente políticos y algunos centros de estudiantes en los que militan miembros de dichos movimientos, que a su vez tienen vínculos con la Federación en las que también hay integrantes de organizaciones políticas. Es decir, los movimientos como la Fuerza Universitaria Rebelde (FUR), Unión Nacional Estudiantil (UNE) e Izquierda autónoma (IA), constituyen nodos que articulan el resto de organizaciones estudiantiles, y su visibilidad está mediada por la obtención de espacios de poder en centros de estudiantes, federación, y representaciones en consejos. Sin embargo, debido a que estas organizaciones representan una proporción minoritaria del estudiantado, la mayor parte de las organizaciones no logran vincularse mucho entre sí, e incluso relatan tener malas experiencias asociadas a las relaciones entre carreras: "Las otras carreras nos tienen mala, en los consejos de carrera se trata como de bajar esa carrera en vez de apoyarla, (...) y eso quizás hace como que ingeniería comercial se aleje un poco" (Presidenta Centro de Estudiantes de Ingeniería Comercial, generación actual).

\subsubsection{VÍNCULOS EXTERNOS A LA UNIVERSIDAD}

En lo relatado por los dirigentes antiguos, la vinculación con organizaciones externas a la universidad, aparece reflejada principalmente la pertenencia a partidos políticos tradicionales, generándose así una concreción local de un proyecto político nacional, dada la verticalidad de los partidos. Los dirigentes actuales en cambio, describen una relación no tan fuerte con las colectividades políticas nacionales, y establecidas principalmente a través de internet. En cambio, relatan desarrollar un conjunto de actividades con agrupaciones 
sociales del entorno, de carácter autogestionado y en algunos casos financiadas con fondos concursables:

Ahora estamos reuniéndonos bastantes chicos que somos estudiantes de pedagogía para trabajar en campamentos de Rahue. (Secretaria de agrupación de estudiantes con hijos, generación actual)

Estamos sacando más proyectos de diferentes índoles, también siguiendo con la robótica y también viendo las necesidades de la comunidad. También estamos sacando un proyecto de alfabetización digital para los abuelitos. (Miembro de Grupo de Robótica, generación actual)

Estas actividades se realizan de forma aislada y tienen en común que son gestadas por parte de estudiantes universitarios para aportar de forma concreta al mejoramiento de las condiciones de vida de grupos de población del entorno de la Universidad. Sin embargo, no constituyen esfuerzos por generar alianzas de largo plazo en el marco de un proyecto político propiamente tal. De manera paralela, las organizaciones como el FUR, la UNE y la IA, tienden a generar discursos en esta línea, pero no alcanzan aún a concretarse.

\subsection{CONTRAPUNTOS TERRITORIALES}

Los relatos de los dirigentes antiguos dan cuenta de un conjunto de particularidades de la dirigencia estudiantil en la ULA, las que asocian a su ubicación territorial respecto de la capital nacional. Plantean que, a pesar de que parte importante de la épica de la época tenía que ver con la recuperación de la democracia al igual que en el resto del país, en el caso específico del IPO y posteriormente la ULA, se generó un debate que no tenía parangón con lo ocurrido en la capital nacional $\mathrm{u}$ otras regiones:

en estas reuniones de la CONFECH (...), lo que nosotros estábamos aceleradamente discutiendo, no estaba ni siquiera en la agenda de 
96 Continuidades y contrapuntos sociopoliticos entre dos generaciones de dirigentes estudiantiles de la Universidad de Los Lagos

preocupación de la mayoría de las dirigencias estudiantiles, entonces yo diría que probablemente que nuestro carácter de Instituto Profesional de Osorno, el estar en el margen del sistema de educación superior impuso una condición como insular en nuestros debates, que le dio a la generación de esa época un sello distintivo. (Presidente Federación de Estudiantes, generación $80-90)$

Este intenso debate estuvo abocado a la conquista de espacios de participación estudiantil en el gobierno universitario, contexto en el que se logró instaurar un modelo de gobierno que ubicó a la ULA en una posición adelantada respecto del escenario nacional en cuanto a participación estudiantil se refiere, condición que perdura hasta hoy:

(la ULA es) la única universidad en la que los estudiantes eligen en un 50\% a sus jefes de carrera, lo que es inédito, no existe en ninguna otra universidad y participen con derecho a voz y voto en todos los cuerpos colegiados de la universidad, que quedó como herencia de esa discusión triestamental. (Presidente Federación de Estudiantes, generación 80-90)

Esta particularidad es valorada también por la generación actual de dirigentes, lo que refuerza la idea anteriormente planteada, de que el hecho de que la actual generación no hace uso de los espacios de participación existentes no se debe a una falta de valoración de ellos, sino que a que el carácter de estos espacios no se adecúa a las actuales exigencias de participación estudiantil en el gobierno universitario. "Yo valoro positivamente los espacios que existen hoy en día de participación al interior de la universidad, hoy en día tenemos la suerte de poder estar en una de las universidades más democráticas del país" (Representante estudiantil en consejo superior, generación actual).

El surgimiento de esta experiencia particular en la región de Los Lagos puede deberse a lo que el presidente de la Federación de la época denomina como condición de insularidad de los debates desarrollados en el proceso de 
transición de IPO a ULA. Es decir, existe una conciencia respecto de la dimensión territorial, especialmente de la distinción entre centro (dirigencias metropolitanas) y periferia (dirigencias regionales-locales). En este sentido, 'insularidad' pudiera entenderse como 'periferialidad' ya que está más a tono con un análisis que releva la intersección entre posiciones sociales y territoriales y, dentro de ello, una distinción entre centro y periferia. Así, mientras insularidad sugiere una idea de separación absoluta, periferia, en cambio, alude a interrelaciones entre ambos locus territoriales. En esta relación mutua entre centro y periferia se producen asimetrías de poder que abren o clausuran la viabilidad de las acciones para uno u otro actor. En este caso, la condición de periferialidad favorece la posibilidad de llevar a cabo experiencias innovadoras que desde el centro no serían tan fáciles de implementar o que implican el riesgo de un fracaso que puede poner en peligro la legitimidad del centro. Con todo, las conquistas logradas por la generación antigua fueron menores a las propuestas planteadas en dicha época. Versiones de gobernanza universitaria con un enfoque de democracia más profunda fueron vetadas desde el nivel nacional.

Una vez que el Instituto Profesional Osorno presentó el proyecto de creación de la universidad tuvo que presentar un estatuto orgánico y ese estatuto orgánico contenía una consideración para que los funcionarios pudieran participar de la elección del rector, lo que fue vetado por el ejecutivo de la época, (...) y por tanto se acomodó el estatuto orgánico a una visión más tradicional, más conservadora de gobernanza universitaria. (Presidente Federación de Estudiantes, generación 80-90)

Por otro lado, la generación actual señala que la condición de periferalidad de la ULA implica que sus organizaciones ocasionalmente encuentran dificultades para vincularse presencialmente con el resto de organizaciones estudiantiles a nivel nacional, aunque ello es en alguna medida mitigado a través de la comunicación por internet: 
98 Continuidades y contrapuntos sociopolíticos entre dos generaciones de dirigentes estudiantiles de la Universidad de Los Lagos

El tema de estar lejos geográficamente con algunas otras agrupaciones, (...) nos dificulta viajar por tema monetario, ese yo creo que es el tema de encontrarnos lejos unos de otros pero sí nos facilita tener comunicación por internet. (Agrupación de estudiantes con hijos, generación actual)

Estas relaciones virtuales a nivel nacional se dan más comúnmente entre las organizaciones como el FUR, UNE e IA, y tienen características marcadamente distintas a las del vínculo entre el gobierno universitario de universidades regionales como la ULA y el gobierno central. Mientras estas últimas son asimétricas, las primeras son horizontales, de acuerdo a lo relatado por los miembros de estas organizaciones.

\section{CONCLUSIONES}

Se realizó un contraste entre dos generaciones de dirigentes estudiantiles de la Universidad de Los Lagos, a partir de sus discursos, analizando tanto cambios como permanencias en cuanto a épica, modos de organización y vínculos tanto internos como externos. Además, se analizaron los contrapuntos que ambas generaciones realizan respecto de la capital nacional.

Los resultados dan cuenta de que las épicas han cambiado. Mientras en la generación de finales de los 80 y principios de los 90, la épica estaba permeada por la lucha contra la dictadura tanto a nivel nacional como en la vida universitaria local, en la generación actual se da más bien una coexistencia de dos lógicas: por un lado, lo que denominamos luchas focalizadas; y, por otro lado, una épica del sujeto político. Es decir, la colectivización de lo individual, lo que concuerda con la idea de que lo que hoy enfatizan las nuevas generaciones es una política enfocada principalmente en el sujeto.

Lo anterior se asocia al hecho ya sabido de la pérdida de legitimidad de los 
partidos políticos tradicionales que fueron relevantes para la generación pasada, pero que en la generación actual han sido reemplazados por movimientos políticos que, en vez de los antiguos modos de organización jerárquicos, establecen relaciones más bien horizontales y de democracia profunda. Sin embargo, a diferencia de lo observado en el pasado, el discurso de estos movimientos ocupa un lugar secundario entre los estudiantes de la universidad. Esto implica que la épica, modos de organización y formas de concebir las vinculaciones internas y externas de estos movimientos, no logran permear el discurso general de las organizaciones estudiantiles, como se observa en los relatos de centros de estudiantes y otras organizaciones sociales de la universidad. Habría que intentar, en un estudio posterior, contrastar esta disociación, según la composición de clase y el capital político y cultural de ambas generaciones de estudiantes.

En cuanto a los modos de organización, destaca la crítica hecha por la generación antigua a la actual, de no valorar los espacios de participación conquistados en el pasado. Sin embargo, este hecho puede ser explicado a raíz de las nuevas exigencias planteadas por la actual generación en relación a la búsqueda de una democracia más profunda, que no encuentra satisfacción suficiente en las instancias formales de participación conquistadas en el pasado. Aunque también debe tenerse en consideración lo planteado por los dirigentes actuales (especialmente aquellos vinculados a movimientos políticos extralocales) en relación al efecto profundo y de largo plazo que ha tenido el modelo neoliberal en la desarticulación de los actores sociales, razón por la cual parte importante de la épica de los movimientos propiamente políticos presentes en la ULA tiene que ver con dinamizar la vida social y política la universidad, ya que aún en las instancias de participación internas de las organizaciones estudiantiles, difícilmente se logra una convocatoria mayoritaria.

La forma de vincularse tanto con actores internos como externos también ha experimentado cambios. La generación pasada transitó desde acciones de 
100 Continuidades y contrapuntos sociopoliticos entre dos generaciones de dirigentes estudiantiles de la Universidad de Los Lagos

presión a una actitud más dialogantes con las autoridades académicas, en tanto que la actual generación no ha vivenciado dicho proceso de igual forma. No obstante, sí se vinculan con autoridades académicas para fines prácticos sin concordar necesariamente en un alcance político de orden más general. Es decir, las vinculaciones de la generación actual tienden a ser más pragmáticas, sin estar en el contexto de un proyecto político propiamente tal. Ello es así también en el ámbito de las vinculaciones con actores externos.

En suma, encontramos que, en el caso analizado, ha habido un cambio generacional relevante, con tendencia a un giro hacia una épica, modos de organización y vinculaciones guiadas por criterios de pragmatismo $\mathrm{y}$ subjetivación, en un contexto de micro-luchas colectivizadas que no se enmarcan en una visión política en común a la manera tradicional y, mucho menos, una que sea viabilizada en términos organizacionales nuevamente tradicionales. Sin embargo, al mismo tiempo se observa la presencia en un plano secundario de una épica orientada a la recomposición del sujeto político, proyecto que por sí mismo empuja los límites de lo que tradicionalmente se entiende como política hacia su dimensión cultural subjetiva. Esta visión se encuentra en los dirigentes pertenecientes a los movimientos políticos extralocales que hoy disputan la legitimidad mermada de los partidos políticos tradicionales.

En relación a los contrapuntos territoriales, se concluye en general que el territorio, en efecto, es productor, reproductor y agente modificador de diversas configuraciones de la política entre las que destacamos la generacional, como lo plantean Vomaro y Daza (2017, p.46). Esto se expresa en diferencias territoriotemporales entre las dos generaciones analizadas. La generación antigua fue protagonista de una iniciativa de vanguardia en el país que aún hoy ubica a la ULA en una posición aventajada en cuanto a democracia interna. Sin embargo, en la actualidad pareciera ser que la condición de periferalidad, otrora vista como una oportunidad para la realización de cambios sin el peso tan fuerte de la 
injerencia del nivel central, hoy es representada más bien como una barrera para el vínculo presencial con otras organizaciones del territorio nacional y, especialmente, del centro.

En este sentido, para las dirigencias actuales, el territorio opera -en palabras de Giménez (1999) - como una especie de prisión originaria, en tanto dificulta una incidencia que vaya más allá de lo meramente local. Esto puede deberse a que, al ya no contar con los partidos políticos tradicionales como medio de socialización y formación política, las nuevas dirigencias de una universidad como la analizada -cuyo estudiantado proviene principalmente de clases sociales populares y de ciudades intermedias (Osorno, Puerto Montt) o territorios rurales o semi rurales aledaños- ve reducidas las posibilidades de incrementar su capital social y cultural e integrarse de forma profunda a circuitos o redes políticas de alcance nacional.

Ahora bien, en términos generales, aunque las diferencias intergeneracionales parecen evidentes, los resultados hacen cuestionarse el rol supuestamente demarcador que, según la literatura revisada, tuvieron las movilizaciones de los años 2006 y 2011 en la generación actual, en el caso de una región alejada del centro nacional como es Los Lagos. Es decir, las movilizaciones masivas llevadas a cabo en esos periodos, aunque sí dan cuenta del cambio cualitativo que socialmente se ha instalado en cuanto al valor de la participación y del sujeto estudiantil movilizado, parecieran no dar cuenta necesariamente de un aumento cuantitativo de la participación estudiantil tanto en las instancias formales del gobierno universitario, como en instancias internas de las organizaciones estudiantiles. Además, el análisis realizado pareciera dar cuenta más bien de un aumento de las distancias entre las dirigencias de la región de Los Lagos versus la capital, en un sentido no solo físico, sino también social, cultural y simbólico. Como plantea Bourdieu, la posición social no solo responde a dimensiones tradicionalmente consideradas, como clase social, sino que también "a una distribución determinada en el 
102 Continuidades y contrapuntos sociopoliticos entre dos generaciones de dirigentes estudiantiles de la Universidad de Los Lagos

espacio geográfico (que nunca es totalmente neutra)" (Bourdieu, 2002, p.100). Al parecer, como plantean Vomaro y Daza (2017) citando a Michel Foucault y Edward Soja, la relevancia de la dimensión espacial en la explicación de los fenómenos sociales ha aumentado en las últimas décadas. Ello queda reflejado en los contrastes territorio-temporales entre las generaciones analizadas.

Otra vía de interpretación posible es que las fuentes de inspiración para una épica de los movimientos estudiantiles contemporáneos (al menos en el caso estudiado) ya no provienen de discursos o proyectos políticos totalizantes, sino más bien de un complejo crisol de inquietudes, intereses y demandas asentados en la sumatoria espontánea de agrupaciones parceladas. En este sentido, se estaría frente a un escenario de profundización en dos direcciones opuestas: una profundización de la subjetivación política como fuente de épicas colectivas y una profundización de la demanda por instancias de democracias más radicales en el gobierno universitario. La coexistencia de dos lógicas aparentemente contradictorias (las luchas focalizadas y la épica del sujeto político), pueden tener que ver con una crisis signada por dos procesos. Por una parte, la persistencia de una sensibilidad sociopolítica caracterizada por la deslegitimación de las épicas totalizantes de décadas previas, y junto con ello la deslegitimación de la práctica política de carácter institucional y partidista de quienes sostenían esos discursos. Es posible que el desanclamiento de esta sensibilidad haya llevado a la lógica de las luchas focalizadas, ya que la política entendida como fenómeno relacional (y no como sustancia) no se pierde, sino que se transforma o se repliega. Por otra parte, la emergencia de un nuevo proyecto sociopolítico, esta vez caracterizado no por la aplicación de un modelo previo a la usanza de las vanguardias, sino por la vitalización de espacios en que pueda emerger el sujeto político. Se trata de un proyecto todavía en ciernes, según se desprende de su carácter "minoritario" como se ha visto en este estudio, y que aún es incipiente en su formulación teórica, especialmente porque opera bajo un principio de dialogicidad y democracia profunda. 
Cómo es posible que ambas dinámicas coexistan, y de qué manera este escenario ha llegado a constituirse resulta un interesante espacio investigativo a recorrer en lo sucesivo.

\section{REFERENCIAS}

Améstica, L., Gaete, H. y Llinas-Audet, X. (2014). Segmentación y clasificación de las universidades en Chile: desventajas de inicio y efectos de las políticas públicas de financiamiento. Ingeniare, revista chilena de ingeniería, 22(3), pp. 384 -397.

Bajoit, G. y Vanhulst, J. (2016). Las acciones colectivas conflictivas: el caso del movimiento estudiantil chileno. Cuadernos de la Escuela de Sociología, UCM, Vol. 1, nº 1 .

Bellei, C. (2013). El estudio de la segregación socioeconómica y académica de la educación chilena. Estudios pedagógicos 39(1), pp. 325-345.

Bergua, J. (2016). Creatividad: Números e imaginarios. Madrid, España: Centro de investigaciones sociológicas.

Bro, N. (2011). Condiciones culturales en la constitución de identidades políticas en el Chile actual. Algunas reflexiones a partir del caso del movimiento estudiantil secundario de 2006. Polis 10(28), 51-68.

Cabalin, C. (2014). Online and mobilized students: the use of Facebook in the Chilean student protests. Comunicar, 43(22), 25-33.

Carrasco, D., Carrasco, J. y Guerra, E. (2014). Juventud y política en Antofagasta: hacia una reinscripción de la experiencia política en jóvenes. Última Década, 22(40), 85-107.

Carrasco, G. (2010). Participación y tendencias políticas en estudiantes universitarios: el caso de la Universidad de Chile. Última Década, 18(32), 85-103.

Castells, M. (2012). Redes de indignación y esperanza: los movimientos sociales en la era de internet. Madrid, España: Alianza.

Cheresky, I. (2006). Elecciones en América Latina: poder presidencial y liderazgo político bajo la presión de la movilización de la opinión pública y la ciudadanía. Nueva Sociedad, 206, pp. 14-26.

Cifuentes, L., Cuenca, J. y Salinas, R. (1998). En defensa de la universidad estatal chilena. Ciencia al Día, 1(1).

Cummings, P. (2015). Democracy and student discontent: Chilean student protest in the post-Pinochet era. Journal of Politics in Latin America, 3, 
104 Continuidades y contrapuntos sociopoliticos entre dos generaciones de dirigentes estudiantiles de la Universidad de Los Lagos

pp. 49-84.

Delamaza, G. (2010). Mapa de actores regionales estratégicos y su articulación con el sector público para la descentralización, en tres regiones de Chile (Informe del estudio "Articulación de Actores para la Descentralización en Tres Regiones de Chile", Subsecretaría de Desarrollo Regional y Administrativo).

Delamaza, G. (2013). Fortalecimiento de las capacidades regionales. Documentos de trabajo RIMISP, $\mathrm{n}^{\circ} 9$.

Delamaza, G. (2013b) De la elite civil a la elite política: Reproducción del poder en contextos de democratización. Polis, 12(36), 67-100.

Donoso, S. (2013). Dynamics of change in Chile: Explaining the emergence of the 2006 Pingüino Movement. Journal of Latin American Studies, 45(1), pp. $1-29$.

Fernández, G. (2000). Notas sobre la participación política de los jóvenes chilenos. En: S. Balardini (comp.), La participación social y politica de los jóvenes en el horizonte del nuevo siglo. Buenos Aires, Argentina: CLACSO, pp. 87-108.

Fleet, N. (2011). Movimiento estudiantil y transformaciones sociales en Chile: una perspectiva sociológica. Polis, 10(30), pp. 99-116.

Garretón, M. (1991). Problemas y desafíos en la participación política de los jóvenes. Santiago, Chile: FLACSO.

Ghiardo, F. (2004). Generaciones y juventud: una relectura desde Manheimm y Ortega y Gasset. Última Década, 20(12), 11-46.

Gravano, A. (2016). Épicas barriales: lo público-político vivo. Cuadernos de Antropología, 26(2), pp. 1-25.

Huneeus, C. (1973). Movimientos universitarios y generación de élites dirigentes: Estudio de casos. Santiago, Chile: Corporación de Promoción Universitaria.

Instituto Nacional de la Juventud [INJUV]. (2004). La integración social de los jóvenes en Chile: 1994-2003. $4^{a}$ Encuesta Nacional del INJUV. Santiago, Chile: INJUV.

Leccardi, C. y Feixa, C. (2011). El concepto de generación en las teorías sobre la juventud. Última Década, 19(34), pp. 11-32.

Lobos, M. (2014). La influencia de las organizaciones políticas universitarias en la formación de élites políticas en Chile: El caso de las federaciones de estudiantes de la Universidad de Chile y Universidad Católica 19842005. Revista de Ciencia Política, 52(2), pp. 157-183.

Mac-Clure, O. y Calvo, R. (2013). Desigualdades sociales y tipos de territorios 
en Chile. Polis, 12(34), pp. 467-490.

Mac-Clure, O. (2012). Las nuevas clases medias en Chile: análisis de cohorte. Revista CEPAL, 108, pp. 169-182.

Madrid, S. (2005). ¿Políticos de ayer, apáticos de hoy? Generaciones, juventud y política en Chile. En: C. Fuentes y A. Villar (eds.), El ciudadano a las urnas: un debate sobre la participación electoral en Chile (pp. 45-84). Santiago, Chile: FLACSO.

Marcel, M., y Tokman, C. (2005). ¿Cómo se financia la educación en Chile? Estudios de Finanzas Públicas n5, Dirección de PresupuestosMinisterio de Hacienda.

Maturana, F. (2015). ¿Ciudad media o ciudad intermedia? Evolución conceptual y estudio en Chile. En: F. Maturana y A. Rojas (ed.), Ciudades intermedias en Chile: Territorios olvidados (pp. 21-42). Santiago, Chile: Ril.

Mayol, A. y Azócar, C. (2011). Politización del malestar, movilización social y transformación ideológica: el caso "Chile 2011". Polis, 10(30), pp. 163184.

Muñoz, V. (2011). Juventud y política en Chile. Hacía un enfoque generacional. Última Década, 19(35), pp. 113-141.

Núñez, J. y Gutiérrez, R. (2004). Class discrimination and meritocracy in the labor market: evidence from Chile. Estudios de Economía, 31(2), pp. 113-132.

OCDE. (2011). Indicadores Sociales, resultados clave de Chile (Society at a Glance 2011). Recuperado desde: http://www.oecd.org/chile/47572883.pdf

Orellana, C., Bivort, B., Farías, F. y Martínez, S. (2014). Jóvenes y participación política: ¿no afección partidista o nuevas ciudadanías? TS Cuadernos de Trabajo Social, 11, pp. 23-33.

Rodríguez, R., Peña, P. y Sáez, Ch. (2014). Crisis y cambio social en Chile (2010-2013): El lugar de los medios de los movimientos sociales y de los activistas digitales. Anagramas, 12(24), pp. 71-94.

Salinas, D. y Fraser, P. (2012). Educational opportunity and contentious politics: The 2011 Chilean Student Movement. Berkeley Review of Education, 3(1), pp. 17-47.

Toro, S. (2008). De lo épico a lo cotidiano: Jóvenes y generaciones políticas en Chile. Revista Ciencia Política, 28(2), pp. 143-160.

Universidad de Los Lagos. (2013). Plan Estratégico de Desarrollo Institucional 2013-2018. 
106 Continuidades y contrapuntos sociopoliticos entre dos generaciones de dirigentes estudiantiles de la Universidad de Los Lagos

Universitas/El Mercurio. (2015). Ranking de calidad de las universidades chilenas. Recuperado desde: http://rankinguniversidades.emol.com/category/rankings/2015/

Valderrama, L. (2013). Jóvenes, ciudadanía y tecnologías de información y comunicación. el movimiento estudiantil chileno. Revista Latinoamericana de Ciencias Sociales, Niñez y Juventud, 11(1), pp. 123-135.

Valenzuela, K. (2007). Colectivos juveniles: ¿inmadurez política o afirmación de otras políticas posibles? Última Década, 15(26), pp. 31-52.

Vomaro, P. y Daza, A. (2017). Politizaciones generacionales y procesos de territorialización en las periferias urbanas: la experiencia del sur del Gran Buenos Aires entre los años ochenta y la actualidad. Última Década, 25(47), pp. 44-82.

Waissbluth, M. (2010). Se acabó el recreo: La desigualdad en la educación. Santiago, Chile: Random House Mondadori.

Zarzuri, R. (2010). Tensiones y desafíos en la participación política juvenil en Chile. Utopía y Praxis Latinoamericana, 15(50), pp. 103-115.

FECHA DE RECEPCIÓN: NOVIEMBRE 2016.

FECHA DE ACEPTACIÓN: NOVIEMBRE 2017. 